\title{
SAFE: A Strategy-Proof Auction Mechanism for Multi-radio, Multi-channel Spectrum Allocation ${ }^{\star}$
}

\author{
Ruihao Zhu, Fan $\mathrm{Wu}^{\star \star}$, and Guihai Chen \\ Shanghai Key Laboratory of Scalable Computing and Systems \\ Shanghai Jiao Tong University, China \\ zhurh1992@sjtu.edu.cn, \{fwu,gchen\}@cs.sjtu.edu.cn
}

\begin{abstract}
The rapid growth of wireless technology has led to increasing demand for spectrum. In the past, spectrum is statically allocated. As a result, many wireless applications cannot use idle spectrum even though it is left unused by the owner for a long period of time. The low utilization of already scarce spectrum resource requires us to dynamically reallocate the idle spectrum to achieve better spectrum usage. In this paper, we model the problem of spectrum reallocation as a sealed-bid reserve auction, and propose SAFE, which is a $\underline{\text { Strategy-proof }}$ Auction mechanism For multi-radio, multi-channel spEctrum allocation. We prove the strategy-proofness of SAFE theoretically, and evaluate its performance extensively. Evaluation results show that SAFE achieve good performance, in terms of spectrum utilization and buyer satisfaction ratio.
\end{abstract}

\section{Introduction}

Radio spectrum is under great demand due to the latest development of wireless technology. The static allocation of radio spectrum can no longer meet the growth of wireless applications since it not only leaves lots of radio spectrum unused in some geographic areas, but also makes the idle radio spectrum unavailable to new wireless applications that do not have licensed spectrum bands. Consequently, dynamic radio spectrum reallocation is highly needed to solve or alleviate the problem of spectrum shortage.

A feasible way to reallocate radio spectrum is to perform auction. The Federal Communications Commission (FCC) has adopted this method for approximately two decades [3]. In contrast to FCC, which only holds auctions for large buyers, we concentrate on small buyers like private wireless networks and mobile wireless applications.

However, there are two major difficulties in designing efficient mechanisms for radio spectrum auction. One difficulty, coming from the auction theory itself, is strategyproofness (see Section 3.2 for the definition of strategy-proofness). Generally speaking,

\footnotetext{
* This work was supported in part by the State Key Development Program for Basic Research of China (Grant No. 2012CB316201), in part by China NSF grant 61272443, 61133006, 61073152, and in part by Shanghai Science and Technology fund 12PJ1404900 and 12ZR1414900. The opinions, findings, conclusions, and recommendations expressed in this paper are those of the authors and do not necessarily reflect the views of the funding agencies or the government.

** Corresponding author.
}

K. Ren et al. (Eds.): WASA 2013, LNCS 7992, pp. 121-134 2013.

(C) Springer-Verlag Berlin Heidelberg 2013 
strategy-proofness means that any buyer cannot get higher payoff by bidding a value other than her true valuation for the goods. In the radio spectrum auction, the buyers are rational and always try their best to maximize their own payoffs. The buyers may manipulate the auction to seek for more benefit, and thus lower the spectrum utilization and hurt the other buyers' interests. As a result, designing a strategy-proof mechanism for the spectrum auction is of undoubted importance. The other difficulty comes from the reusability of the radio spectrum. The reusability of the radio spectrum can allow two buyers to use the same spectrum simultaneously as long as they have enough distance (out of the interference range) between each other geographically. What's more, the optimal solution for the problem of spectrum allocation is NP-complete [1, 19] in multi-hop wireless networks.

In the past, several works on strategy-proof auction mechanisms for spectrum allocation have been proposed [12], For example, Zhou et al.'s VERITAS [20] and TRUST [21], Wu and Vaidya's SMALL [15]. VERITAS uses the method of critical neighbor to solve a single side radio spectrum auction. However, it does not take the valuation of the sellers into account and the seller's utility might be negative. TRUST uses McAfee mechanism to perform a double side auction and it could guarantee the profit of the sellers, but it has to waste a channel and sacrifice a lot of good buyers. SMALL introduces the concept of reserve price to guarantee the profit of seller, and would only sacrifice a bounded number of buyers. However, when applied to multi-radio, multi-channel spectrum auction, SMALL can only work under a very strong assumption that the channels must be sold out.

The existing problems of the previous mechanisms prompt us to design an auction mechanism which can allocate spectrum efficiently in general situations and protect the

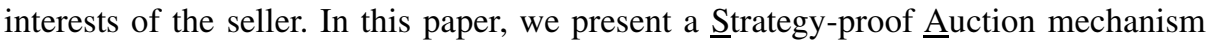
For multi-radio, multi-channel spEctrum allocation (SAFE). In SAFE, there is a seller with multiple radio spectrum channels and has a reserve price for each of the channel. All buyers submit their sealed bids at the beginning of the auction so that they do not have any information about the others' bids. The channel can be reused and each buyer can get more than one channel. The auction mechanism selects the winning buyers and allocates channels to them iteratively. The seller may not sell a channel if the bid for this channel is lower than the reserve price. The major advantages of SAFE are listed as follow:

- SAFE enables a multi-radio, multi-channel spectrum auction, in which all the buyers can bid for more than one channel and get more than one channel as well.

- When compared with VERITAS, SAFE has higher spectrum utilization and buyer satisfaction ratio, which are two commonly used metrics for evaluating channel auction mechanisms. Spectrum utilization is the sum of the number of channels that the buyers get divided by the number of channels; and the buyer satisfaction ratio is the proportion of the buyers who gets at least a given ratio of their requested channels.

- When compared with TRUST, the seller can possibly sell all the channels; while in TRUST, the seller always has to waste one channel. SAFE sacrifices much less buyers than TRUST, and the number of sacrificed buyers by SAFE is bounded by the number of channels. 
- When compared with SMALL, SAFE does not depend on the very strong assumption that the channels must be sold out.

The major contributions in this paper are as follows: We propose a strategy-proof auction mechanism for multi-radio, multi-channel spectrum auction, namely SAFE, and prove its strategy-proofness. We also implement SAFE, and numerically evaluate its performance. We list our detailed contributions as follows:

- Strategy-Proofness: We prove that SAFE can achieve both incentive-compatibility and individual rationality. When SAFE is applied, the dominant strategy of each buyer is to bid her true valuation, and none of them is charged higher than her true valuation for a channel when bidding truthfully.

- Seller Incentives: SAFE adopts the concept of reserve price, and thus guarantees that the seller is profitable by selling her channels.

- Extensive Evaluation: We conduct extensive simulations to evaluate SAFE's performance. The results verify that SAFE does guarantee strategy-proofness, and achieves good performance in terms of spectrum utilization and buyer satisfaction ratio.

The rest of this paper is organized as follows. In Section 2, we review related works. In Section 3, we present technical preliminaries. In Section 4, we introduce the multiradio, multi-channel spectrum auction mechanism-SAFE, and prove that it is a strategyproof mechanism. In Section 5, we give the evaluation results of SAFE. In Section 6 , we draw conclusions and point out possible future work directions.

\section{Related Works}

In this section, we briefly review existing works on radio spectrum auctions.

Zhou et al. proposed VERITAS [20], which uses critical price to determine the charges for the channel winners. Although VERITAS can be applied to multi-radio, multi-channel auction, it suffers from low spectrum utilization due to the nature of the greedy channel allocation. Later, they proposed TRUST [21], which is an elegant double side mechanism adopting the thoughts from McAfee mechanism. Wu and Vaidya have proposed SMALL [15], which incorporates the concept of reserve price to protect the benefit of the channel seller, and achieves superior performance to TRUST. Although SMALL is the closest work to ours, it cannot be applied to general multi-radio, multi-channel auction, because it relies on the assumption that the channels are scarce compared with the users. In contrast to the above works, SAFE can guarantee strategyproofness for multi-radio, multi-channel auction in the general case, and achieve high spectrum utilization and buyer satisfaction ratio.

Online spectrum auction is also an important topic of spectrum allocation. Hajiaghayi et al. [8] studied the supply-limited online auction model and proposed a value- and time-strategy-proof mechanism, which can achieve constant efficiency and revenue-competitiveness. Hajiaghayi et al. [7] also provided a characterization for truthful online auction rules. Later, $\mathrm{Xu}$ et al. [17] proposed an efficient online spectrum auction mechanism that can decide whether to permit each user's exclusive usage of 
spectrum and calculate their corresponding charges. Deek et al. [2] presented Topaz that can allocate spectrum efficiently and achieve strategy-proofness.

There are also some existing game theory based works on spectrum allocation that are not based on auction [6, 18]. Felegyhazi et al. [4] studied Nash equilibria in a static multi-radio, multi-channel allocation game, and proposed two algorithms to converge the system to the Nash equilibria. Later, Wu et al. [16] presented a stronger mechanism for the above problem to make the system converge to a stable state in a single step.

\section{Technical Preliminaries}

In this section, we present our auction model for multi-radio, multi-channel spectrum allocation, and briefly review some important solution concepts from mechanism design.

\subsection{Auction Model of Spectrum Allocation}

We consider a secondary spectrum market. There is a "seller", who is a wireless communication infrastructure provider. She has a number of channels, and wants to sell her idle channels to wireless service providers who do not have official licences on radio spectrum from the government. The wireless service providers are called the "buyers", and want to buy the licenses of the idle channels from the seller to provide services to their customers. We model the problem as a sealed-bid reserve auction. The seller sets a reserve price for each channel for sale. The reserve price of a channel can be regarded as the maintaining cost of the channel. The buyers submit their sealed bids simultaneously, such that the buyers cannot know each other's bid. If the bid (or the group bid from a group of buyers) for a channel is less than the channel's reserve price, the seller can refuse to sell this channel.

In the auction, the seller can acts as the auctioneer, if she is trustworthy; otherwise, a trusted central authority is required to perform as the auctioneer. The seller has a set of orthogonal channels for sale, denoted by $\mathbb{C}=\left\{c_{1}, c_{2}, \ldots, c_{m}\right\}$. As we mentioned, the seller has a reserve price $s_{k}$ for each channel $c_{k} \in \mathbb{C}$. We denote the profile of reserve prices by

$$
s=\left(s_{1}, s_{2}, \ldots, s_{m}\right) .
$$

Each channel can be used by more than one buyers, if they are out of the interference range of each other.

We denote the set of buyers by $\mathbb{N}=\{1,2, \ldots, n\}$. We assume that the buyers do not have preference over the channels. Each buyer $i \in \mathbb{N}$ has a per-channel valuation $v_{i}$, which is private information of the buyer. This is commonly known as type in the literatures. The valuation for a channel can be the revenue gained from providing wireless services using the channel. In the auction, the buyers choose their bids, denoted by

$$
\boldsymbol{b}=\left(b_{1}, b_{2}, \ldots, b_{n}\right),
$$

which are based on their types, and simultaneously submit the sealed bids to the auctioneer. If a buyer $i \in \mathbb{N}$ is equipped with multiple radio interfaces, she can also claim 
to request up to $r_{i}$ channels. In contrast to the per-channel valuation, we assume that the buyers do not cheat about the maximal number of channels they want. This assumption is based on the fact that the buyers do not have the incentives to cheat the maximal number of requested channels. On one hand, if a buyer requests more than enough channels, she may need to pay for extra channels that are not needed at all. On the other hand, if a buyer under claims the number, she definitely cannot win the expected number of channels in the auction. We denote the profile of channel requests by

$$
\boldsymbol{r}=\left(r_{1}, r_{2}, \ldots, r_{n}\right) .
$$

The buyers also submit their channel requests together with the bids to the auctioneer.

The auction mechanism determines the set of winning buyers, channel allocation, and charges for the winners. Let's denote the set of channels won by buyer $i \in \mathbb{N}$ by $A_{i} \subseteq \mathbb{C}$, and the charge for buyer $i$ on each channel $c_{k} \in A_{i}$ by $p_{i}^{k}$. We can denote the channel allocation by

$$
\boldsymbol{A}=\left(A_{1}, A_{2}, \ldots, A_{n}\right),
$$

where $\forall i \in \mathbb{N}, 0 \leq\left|A_{i}\right| \leq r_{i}$. We now can define the utility $u_{i}$ of each buyer $i \in \mathbb{N}$ to be the difference between her total valuation and total charge on the channels won:

$$
u_{i}=v_{i}\left|A_{i}\right|-\sum_{k \in A_{i}} p_{i}^{k}
$$

Clearly, if a buyer does not win any channel in the auction, then both her charge and utility are zero.

We assume that the buyers are rational, and their objectives are to maximize their own utilities. We also assume that the auction is collusion-free.

In contrast, the objective of the auction mechanism is to increase spectrum utilization and buyer satisfaction ratio. Here, spectrum utilization is the sum of channels the winning buyers get diveded by the number of channels $\frac{\sum_{k=1}^{n}\left|A_{k}\right|}{m}$; buyer satisfaction ratio is the proportion of buyers who get at least a given ratio of their requested channels in the auction.

\subsection{Solution Concepts}

In this section, we introduce the solution concepts used in this paper from mechanism design.

A strong solution concept from mechanism design is dominant strategy.

Definition 1 (Dominant Strategy [5]10]). Strategy $a_{i}$ is a player i's dominant strategy, if for any $a_{i}^{\prime} \neq a_{i}$ and any strategy profile of the other players $a_{-i}$,

$$
u_{i}\left(a_{i}, a_{-i}\right) \geq u_{i}\left(a_{i}^{\prime}, a_{-i}\right) .
$$

Intuitively, a dominant strategy of a player is one that maximizes her utility, regardless of what strategies the other players choose. In our sealed-bid reserve auction for spectrum allocation, the strategy of a buyer $i \in \mathbb{N}$ is her bid $b_{i}=a_{i}\left(v_{i}\right)$. 
The concept of dominant strategy is the basis of incentive-compatibility, which means that there is no incentive for any player to lie about her private information, and thus revealing truthful information is the dominant strategy for every player. A company concept is individual-rationality, which means that for every player who faithfully participate the game/auction is expected to gain no less utility than staying outside. We now can introduce the definition of Strategy-Proof Mechanism.

Definition 2 (Strategy-Proof Mechanism [9] [11]). A mechanism is strategy-proof when it satisfies both incentive-compatibility and individual-rationality.

\section{SAFE}

In this section, we present the design of SAFE, and prove its strategy-proofness.

\subsection{Design of SAFE}

When designing SAFE, we follow the design rational of SMALL, but with significant differences. SAFE contains three algorithms: buyer grouping, winner selection, and charge determination.

\section{(1) Buyer Grouping}

SAFE first constructs a conflict graph of the buyers. In the conflict graph, each node represents a buyer. Any pair of buyers who lie within the interference range of each other are connected by an edge in the conflict graph. SAFE then groups the buyers using an existing graph coloring algorithm (e.g., [14]), so that no buyer can be assigned to more than one group and the buyers who are connected are not in the same group. Since the grouping algorithm is bid-independent, no buyer can manipulate this process. We denote the buyer groups by

$$
\mathbb{G}=\left\{g_{1}, g_{2}, \ldots, g_{q}\right\}
$$

where

$$
\begin{gathered}
g_{j} \cap g_{l}=\emptyset, \forall g_{j}, g_{l} \in \mathbb{G}, \\
\text { and } \bigcup_{g_{j} \in \mathbb{G}} g_{j}=\mathbb{N} .
\end{gathered}
$$

We regard each group as a super buyer, and define a group bid for each group $g_{j} \in G$ as:

$$
B_{j}=\left(\left|g_{j}\right|-1\right) \cdot \min \left\{b_{i} \mid i \in g_{j}\right\} .
$$

If more than one buyer report the smallest bid in the group, we regard the one with the smallest alphabetical order as the smallest-bid bidder. We denote the smallest-bid buyer in group $g_{j}$ by $S M A L L E S T\left(g_{j}\right)$. We now get a profile of group bids:

$$
\boldsymbol{B}=\left(B_{1}, B_{2}, \ldots, B_{q}\right) .
$$




\section{(2) Winner Determination}

SAFE determines the winners and channel allocation iteratively. In each iteration, SAFE sorts the remaining channels by the reserve price in a non-decreasing order, and sorts the remaining buyer groups by the group bids in a non-increasing order:

$$
\begin{gathered}
\mathcal{C}^{t}: c_{1}^{t}, c_{2}^{t}, \ldots, c_{m^{t}}^{t}, \quad \text { s.t., } s_{1}^{t} \leq s_{2}^{t} \leq \ldots \leq s_{m^{t}}^{t}, \\
\mathcal{G}^{t}: g_{1}^{t}, g_{2}^{t}, \ldots, g_{q}^{t}, \quad \text { s.t., } B_{1}^{t} \geq B_{2}^{t} \geq \ldots \geq B_{q}^{t} .
\end{gathered}
$$

Here, $t$ indicates that this is in the $t$ th iteration, and $m^{t}$ denotes the current number of remaining channels. Since the number of groups does not change in different iterations, we can use the same $q$ in all the iterations. If two channels have the same reserve price or two groups have the same group bid, the order between them is determined following the alphabetical order.

Next, SAFE finds the maximal possible number of trades $l^{t}$ in the $t$ th iteration, s.t.

$$
\sum_{i=1}^{l^{t}} s_{i}^{t} \leq \sum_{i=1}^{l^{t}} B_{i}^{t}
$$

Finally, SAFE selects the first $l^{t}$ groups in list $\mathcal{G}^{t}$ as winning buyer groups, and assigns the first $l^{t}$ channels in list $\mathcal{C}^{t}$ to the corresponding winner groups. In each winning group, the buyer(s), except the one who bids the smallest in the group, are winning buyers and can get the channel assigned to the group. We denote the set of winning buyers in the $t$ th iteration by

$$
W^{t}=\bigcup_{j=1}^{l^{t}}\left\{i \mid i \in g_{j}^{t} \wedge i \neq S M A \operatorname{LLEST}\left(g_{j}^{t}\right)\right\} .
$$

For each winning buyer $i \in W^{t}$, SAFE decreases its number of requested channels $r_{i}$ by 1 . If $r_{i}=0$, then buyer $i$ 's demand is fully filled, and SAFE removes buyer $i$ from the buyer group $g_{j}$ she belongs to and updates group $g_{j}$ 's group bid:

$$
\begin{gathered}
g_{j}=g_{j} \backslash\{i\} \\
B_{j}=\left(\left|g_{j}\right|-1\right) \cdot \min \left\{b_{i} \mid i \in g_{j}\right\} .
\end{gathered}
$$

SAFE also deletes the channels, which have already been sold, from the set of channels.

SAFE repeats the above procedure until no more winner can be generated (i.e., $l^{t}=0$ ).

The pseudo-code of above winner selection and channel allocation is shown by Algo-

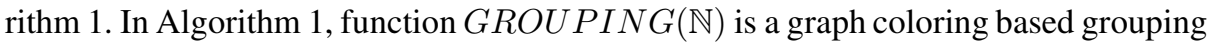
algorithm, and returns the buyer grouping result.

We note that in each trade, only one buyer, who bids the smallest in her buyer group, is sacrificed. As a result, the number of buyers sacrificed does not exceed $m$, which is the number of channels for sale.

Let $d$ denote the largest degree in the conflict graph of the buyers. The computational complexity of the greedy graph coloring based buyer grouping algorithm is $O(n+|E|)$, and the number of groups is at most $(d+1)$. In each iteration, SAFE takes 


\section{Algorithm 1. Winner Determination and Channel Allocation Algorithm}

Require: A set of channels $\mathbb{C}$, a profile of reserve prices $s$, the number of channels $m$, a set of buyers $\mathbb{N}$, a profile of bids $\boldsymbol{b}$, and a profile of channel requests $\boldsymbol{r}$.

Ensure: A set of winning buyers $\mathbb{W}$ and a profile of channel allocation $\boldsymbol{A}$.

$1: \mathbb{W} \leftarrow \varnothing ; \boldsymbol{A} \leftarrow \varnothing^{n} ; t \leftarrow 1 ; m^{t} \leftarrow m$.

2: $(\mathbb{G}, q) \leftarrow G R O U P I N G(\mathbb{N})$.

3: repeat

4: $\quad$ for all $g_{j} \in \mathbb{G}$ do

5: $\quad B_{j}=\left(\left|g_{j}\right|-1\right) \cdot \min \left\{b_{i} \mid i \in g_{j}\right\}$.

6: end for

7: $\quad$ Sort the channels $\mathbb{C}$ by reserve price $s$ in non-decreasing order: $c_{1}^{t}, c_{2}^{t}, \ldots, c_{m^{t}}^{t}$, s.t., $s_{1}^{t} \leq$ $s_{2}^{t} \leq \ldots \leq s_{m^{t}}^{t}$.

8: $\quad$ Sort buyer groups $\mathbb{G}$ by group bid $\boldsymbol{B}$ in non-increasing order: $g_{1}^{t}, g_{2}^{t}, \ldots, g_{q}^{t}$, s.t., $B_{1}^{t} \geq$ $B_{2}^{t} \geq \ldots \geq B_{q}^{t}$.

9: $\quad l^{t} \leftarrow \underset{l^{t} \leq \min \left\{m^{t}, q\right\}}{\operatorname{argmax}}\left(\sum_{i=1}^{l^{t}} s_{i}^{t} \leq \sum_{i=1}^{l^{t}} B_{i}^{t}\right)$.

10: $W^{t} \leftarrow \bigcup_{j=1}^{l^{t}}\left\{i \mid i \in g_{j}^{t} \wedge i \neq S M A L L E S T\left(g_{j}^{t}\right)\right\}$.

11: $\mathbb{W} \leftarrow \mathbb{W} \cup W^{t}$.

12: $\quad$ for $j \leftarrow 1$ to $l^{t}$ do

13: $\quad$ for all $i \in g_{j}^{t} \backslash\left\{S M A L L E S T\left(g_{j}^{t}\right)\right\}$ do

14: $\quad A_{i} \leftarrow A_{i} \cup\left\{c_{j}^{t}\right\} ; r_{i} \leftarrow r_{i}-1$.

15: $\quad$ if $r_{i}=0$ then

16: $\quad g_{j}^{t} \leftarrow g_{j}^{t} \backslash\{i\}$.

17: $\quad$ end if

18: $\quad$ end for

19: $\quad \mathbb{C} \leftarrow \mathbb{C} \backslash\left\{c_{j}^{t}\right\}$.

20: end for

21: $\quad m^{t+1} \leftarrow m^{t}-l^{t} ; t \leftarrow t+1$.

22: until $l^{t}=0$.

23: return $\mathbb{W}$ and $\boldsymbol{A}$.

$\max \{O(m \log m, O(d \log d))\}$ time to sort the bids and reserve price and $O(m)$ time to determine the number of good trades. What's more, SAFE can run at most $m$ iterations. Therefore, the overall computational complexity of SAFE is $O(n+|E|+m$. $\max \{m \log m, d \log d\})$.

\section{(3) Charging}

For each channel $k \in A_{i}$ a buyer $i \in g_{j}$ wins, the charge $p_{i}^{k}$ is equal to the smallest bid in $g_{j}$. So the total charge for a buyer $i \in g_{j}$ is:

$$
p_{i}=\sum_{k \in A_{i}} p_{i}^{k}=\left|A_{i}\right| \cdot \min \left\{b_{k} \mid k \in g_{j}\right\}
$$

We note that the charge for each buyer is independent of her bid.

The seller receives all the charges from the winning buyers. We assume that Algorithm 1 iterates for $\eta$ times. Then the total revenue of the seller is: 


$$
\begin{aligned}
\text { Revenue } & =\sum_{t=1}^{\eta} \sum_{j=1}^{l_{t}}\left(\left|g_{j}^{t}\right|-1\right) \cdot \min \left\{b_{i} \mid i \in g_{j}^{t}\right\} \\
& =\sum_{t=1}^{\eta} \sum_{j=1}^{l_{t}} B_{j}^{t} .
\end{aligned}
$$

From Inequation (1) and Equation (2), we get that the seller's profit is always nonnegative in the auction:

$$
\begin{aligned}
\text { Profit } & =\text { Revenue }-\sum_{t=1}^{\eta} \sum_{j=1}^{l^{t}} s_{j}^{t} \\
& =\sum_{t=1}^{\eta} \sum_{j=1}^{l_{t}} B_{j}^{t}-\sum_{t=1}^{\eta} \sum_{j=1}^{l^{t}} s_{j}^{t} \\
& \geq 0 .
\end{aligned}
$$

\subsection{Strategy-Proofness}

In order to show that SAFE is a strategy-proof auction mechanism for multi-radio, multi-channel spectrum allocation, we prove the following lemma first.

Lemma 1. If SAFE is used, each buyer's dominant strategy is reporting her per-channel valuation as a bid.

Proof. We prove this by showing that by bidding untruthfully, any buyer cannot get a higher utility in any iteration. We consider a buyer $i \in g_{j}$ with per-channel valuation $v_{i}$. Let $b_{j}^{\min }=\min \left\{b_{i} \mid i \in g_{j}\right\}$. Since the smallest-bidding buyer in group $g_{j}$ cannot win any channel, $b_{j}^{\text {min }}$ remains the same in all the iterations. Consequently, buyer $i$ 's utility $u_{i}^{t}$ in the $t$ th iteration is either $v_{i}-b_{j}^{\text {min }}$ or 0 , depending on whether she wins a channel or not in this iteration. Let $\hat{u}_{i}^{t}$ be the utility got by buyer $i$ in the $t$ th iteration, when she bids truthfully. We next prove that buyer $i$ cannot increase her utility got in any iteration, by distinguishing two cases:

1. Buyer $i$ is the smallest-bidding buyer in group $g_{j}$, when bidding truthfully (i.e., $\left.b_{i}=v_{i}\right)$. Then, her utility $\hat{u}_{i}^{t}=0, \forall t$. We further distinguish two cases:

- If she increases her bid to $b_{i}^{\prime}>v_{i}$, then the smallest bid in group $g_{j}$ becomes $b_{j}^{\prime \text { min }} \geq b_{j}^{\text {min }}=v_{i}$. If she still does not win a channel, her utility remains 0 :

$$
u_{i}^{\prime t}=0=\hat{u}_{i}^{t} .
$$

If she wins a channel in the $t$ th iteration, then her utility for this iteration becomes non-positive:

$$
u_{i}^{\prime t}=v_{i}-b_{j}^{\prime m i n} \leq v_{i}-v_{i}=0 .
$$


- If she decreases her bid to $b_{i}^{\prime}<v_{i}$, then she is still the smallest-bidding buyer in group $g_{j}$, and her utility remains 0 .

2. Buyer $i$ is not the smallest-bidding buyer in group $g_{j}$, when bidding truthfully. We further distinguish three cases:

- If she increases her bid to $b_{i}^{\prime}>v_{i}$, then the smallest bid in group $g_{j}$ remains unchanged. Consequently, her utility also remains unchanged, no matter she wins a channel or not in this iteration.

- If she decreases her bid to $b_{i}^{\prime}<v_{i}$ and becomes the smallest-bidding buyer in the group $g_{j}$, then she definitely cannot win a channel and her utility is $u_{i}^{\prime t}=0 \leq \hat{u}_{i}^{t}$.

- If she decreases her bid to $b_{i}^{\prime}<v_{i}$ but is still not the smallest-bidding buyer in the group $g_{j}$, then the group bid of $g_{j}$ remains unchanged. No matter the group $g_{j}$ is a winning group in the $t$ th iteration or not, the buyer $i$ 's utility remains unchanged:

$$
\begin{cases}u_{i}^{\prime t}=v_{i}-b_{j}^{\text {min }}=\hat{u}_{i}^{t}, & g_{j} \text { is a winning group } \\ u_{i}^{\prime t}=0=\hat{u}_{i}^{t}, & \text { otherwise. }\end{cases}
$$

Since buyer $i$ maximizes her utility gain in each iteration by bidding truthfully (i.e., $b_{i}=v_{i}$ ), and the total utility of buyer $i$ is the sum of her utilities gained in all the iterations, we can conclude that buyer $i$ maximizes her utility by bidding truthfully. This completes our proof.

\section{Lemma 2. SAFE guarantees the individual rationality.}

Proof. By the charging scheme, for each channel won, a buyer $i \in g_{j}$ is not charged more than her bid. If the buyer $i$ bids truthfully, then the per-channel charge is also no larger than her per-channel valuation:

$$
\begin{aligned}
\hat{u}_{i} & =v_{i}\left|A_{i}\right|-\sum_{k \in A_{i}} p_{i}^{k} \\
& =\left|A_{i}\right|\left(v_{i}-\min \left\{b_{k} \mid k \in g_{j}\right\}\right) \\
& \geq 0 .
\end{aligned}
$$

Since SAFE satisfies both incentive compatibility and individual rationality, we have the following theorem.

Theorem 1. SAFE is a strategy-proof auction mechanism multi-radio, multi-channel spectrum allocation.

\section{Numerical Results}

We implement SAFE and evaluate its performance in terms of buyer utility, spectrum utilization and buyer satisfaction ratio. 


\subsection{Methodology}

We run SAFE for over 1000 times to evaluate its performance. The terrain area is 1800 meters $\times 1800$ meters, and the buyers are randomly distributed in this area. The number of buyers varies from 50 to 800 with step of 50 . The number of channels is 12 or 24 , and each buyer can request up to 5 channels. The interference range of the buyers is 425 meters. The reserve prices of the channels lie in the range of $(0,1]$, and the buyers' per-channel valuations also lie in the same range $(0,1] 1$ We group the buyers using a greedy graph coloring algorithm [13].

\subsection{Metrics}

We use three metrics to measure SAFE's performance:

- Buyer Utility: For each buyer, her utility is the difference between her total valuation and total charge on the channels won. We distinguish two kinds of buyer behaviors that may result in different buyer utilities.

- Bidding Truthfully: Bidding truthfully means that the bid submitted by a buyer is her true per-channel valuation.

- Misreporting: Misreporting means that a buyer submits a bid other than her true per-channel valuation. In the evaluation, we assume that a misreported bid also lies in $(0,1]$.

- Spectrum Utilization: Spectrum utilization is the sum of the number of channels that the buyers get divided by the number of channels.

- Buyer Satisfaction Ratio: Buyer satisfaction ratio is defined as the proportion of the buyers who get at least a given ratio of their requested channels.

Since TRUST and SMALL cannot guarantee strategy-proofness for multi-radio, multichannel spectrum auction in general cases, we only compare the performance of SAFE with VERITAS.

\subsection{Buyer Utility}

We numerically verify the strategy-proofness of SAFE with 500 buyers and 24 channels. We randomly choose a buyer and investigate her utilities with different behaviors. We run SAFE for over 1000 times. In each run, we fix the other buyers' bids, and evaluate the chosen buyer's utilities of truthfully bidding and misreporting. After that, we randomly choose 50 records to show. We note that the rest records lead to the same conclusion.

Fig. 1] shows the utilities of buyer 178 with different node behaviors. According to the figure, we can find that buyer 178's utility of bidding truthfully is always at least as high as that of misreporting in each run. Furthermore, her utilities of bidding truthfully are always nonnegative, while misreporting can even lead to negative utilities (e.g., utility of misreporting is negative in the 20th, 33rd and 46th run).

\footnotetext{
${ }^{1}$ The ranges of buyers' per-channel valuations and seller's reserve prices can be chosen differently from the ones used here. However, the evaluation results of using different ranges are similar to each other. As a result, we only show the results for the above ranges in this paper.
} 


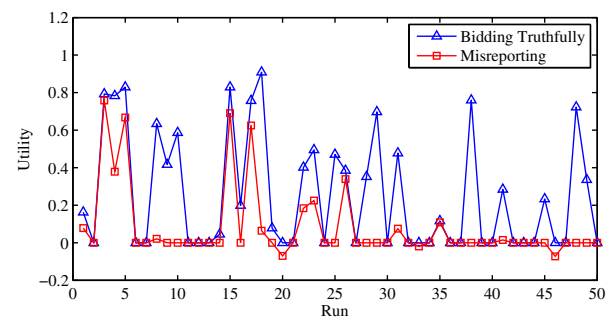

Fig. 1. Utilities of buyer 178 when bidding truthfully and misreporting

\subsection{Spectrum Utilization}

In this subsection, we show the comparison results on spectrum utilization between SAFE and VERITAS.

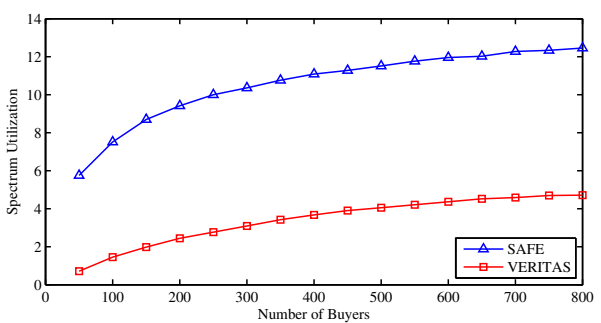

(a) 12 Channels

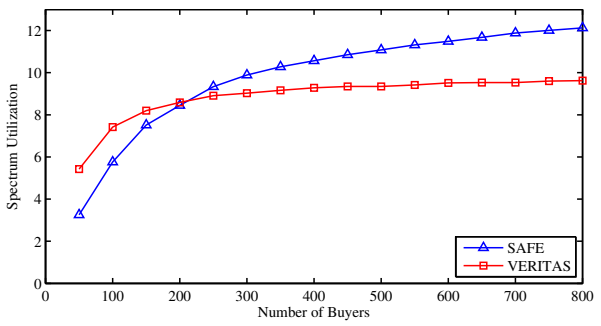

(b) 24 Channels

Fig. 2. Spectrum utilization of SAFE and VERITAS

Fig. 2(a) and Fig. 2(b) show the comparison results on spectrum utilization of SAFE and VERITAS with 12 and 24 channels, respectively. The results show that SAFE outperforms VERITAS in terms of spectrum utilization in most of the situations. The only exception is when there are 24 channels and the number of buyers is small (i.e., less than 200). This is because when the total number of channels requested by the buyers is relatively small compared with the number of channels, VERITAS can possibly fill large proportion of the buyers' request. The results also prove that SAFE is suitable for the situations in which spectrum is a scarce resource. 


\subsection{Buyer Satisfaction Ratio}

Finally, we compare the buyer satisfaction ratios of SAFE and VERITAS. Here, for fair comparison, we set the threshold ratio to be 0.5 , which means that a buyer in SAFE is satisfied if she gets at least $50 \%$ of her requested channels.

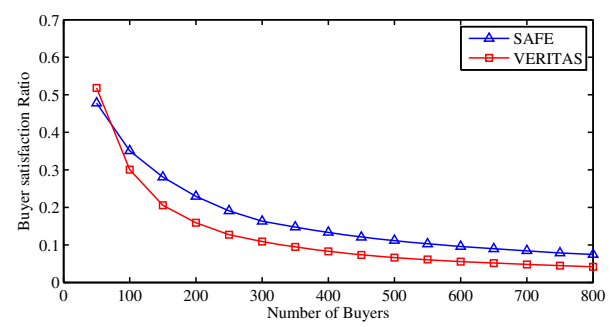

(a) 12 Channels

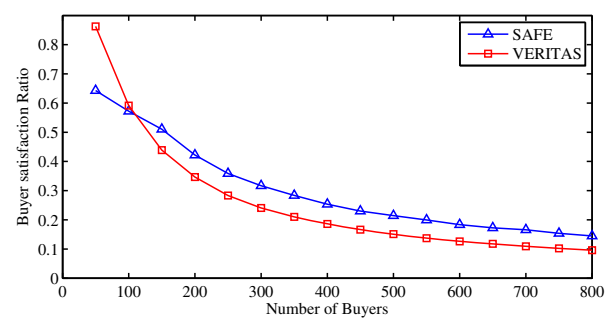

(b) 24 Channels

Fig. 3. Buyer satisfaction ratio of SAFE and VERITAS

Fig. 3(a) and Fig. 3(b) show the comparison results on buyer satisfaction ratios of SAFE and VERITAS with 12 and 24 channels, respectively. From the figures, the results show that SAFE outperforms VERITAS in terms of buyer satisfaction ratio in most of the cases. The only exception is when the number of buyers is small (i.e., less than 100 buyers for 12 channels and less than 150 buyers for 24 channels), VERITAS has a higher buyer satisfaction ratio. This is because SAFE has to sacrifice the buyers who bid the least in the buyer groups to guarantee strategy-proofness.

From the above results, we can draw the conclusion that SAFE is an efficient strategyproof auction mechanism for multi-radio, multi-channel spectrum allocation.

\section{Conclusions and Future Work}

In this paper, we have modeled the problem of multi-radio, multi-channel spectrum allocation as a sealed-bid auction. We have proposed SAFE, which achieves both strategyproofness and high system performance, in terms of spectrum utilization and buyer satisfaction radio. As for future work, we are going to design collusion-resistant auction mechanisms for the multi-radio, multi-channel spectrum allocation. 


\section{References}

1. Cox, D.C., Reudink, D.O.: Dynamic channel assignment in high capacity mobile communication system. Bell System Technical Journal 50(6), 1833-1857 (1971)

2. Deek, L., Zhou, X., Almeroth, K., Zheng, H.: To preempt or not: Tackling bid and time-based cheating in online spectrum auctions. In: INFOCOM 2011 (April 2011)

3. Federal Communications Commission (FCC), http://www. fCC.gov/

4. Félegyházi, M., Čagalj, M., Bidokhti, S.S., Hubaux, J.-P.: Non-cooperative multi-radio channel allocation in wireless networks. In: INFOCOM 2007 (May 2007)

5. Fudenberg, D., Tirole, J.: Game Theory. MIT Press (1991)

6. Gao, L., Wang, X.: A game approach for multi-channel allocation in multi-hop wireless networks. In: MobiHoc 2008 (December 2008)

7. Hajiaghayi, M.T.: Online auctions with re-usable goods. In: EC 2005 (2005)

8. Hajiaghayi, M.T., Kleinberg, R., Parkes, D.C.: Adaptive limited-supply online auctions. In: EC 2004 (2004)

9. Mas-Colell, A., Whinston, M.D., Green, J.R.: Microeconomic Theory. Oxford Press (1995)

10. Osborne, M.J., Rubenstein, A.: A Course in Game Theory. MIT Press (1994)

11. Varian, H.: Economic mechanism design for computerized agents. In: USENIX Workshop on Electronic Commerce (1995)

12. Wang, X., Li, Z., Xu, P., Xu, Y., Gao, X., Chen, H.: Spectrum sharing in cognitive radio networks - an auction based approach. IEEE Transactions on System, Man and CyberneticsPart B: Cybernetics 40, 587-596 (2010)

13. Welsh, D.J.A., Powell, M.B.: An upper bound for the chromatic number of a graph and its application to timetabling problems. The Computer Journal 10(1), 85-86 (1967)

14. West, D.B.: Introduction to Graph Theory, 2nd edn. Prentice Hall (1996)

15. Wu, F., Vaidya, N.: SMALL: A strategy-proof mechanism for radio spectrum allocation. In: INFOCOM 2011 (April 2011)

16. Wu, F., Zhong, S., Qiao, C.: Globally optimal channel assignment for non-cooperative wireless networks. In: INFOCOM 2008 (April 2008)

17. Xu, P., Xu, X., Tang, S., Li, X.-Y.: Truthful online spectrum allocation and auction in multichannel wireless networks. In: INFOCOM 2011 (April 2011)

18. Yu, Q., Chen, J., Fan, Y., Shen, X.S., Sun, Y.: Multi-channel assignment in wireless sensor networks: A game theoretic approach. In: INFOCOM 2010 (April 2010)

19. Yue, W.: Analytical methods to calculate the performance of a cellular mobile radio communication system with hybrid channel assignment. IEEE Transactions on Vehicular Technology 40(2), 453-460 (1991)

20. Zhou, X., Gandhi, S., Suri, S., Zheng, H.: eBay in the sky: Strategy-proof wireless spectrum auctions. In: MobiCom 2008 (September 2008)

21. Zhou, X., Zheng, H.: TRUST: A general framework for truthful double spectrum auctions. In: INFOCOM 2009 (April 2009) 